$\$$

\title{
Solving Richards' nonlinear PDE modeling dynamic from water in unsaturated zone by a new numerical method called SBA
}

\author{
Ben-Sthal Sakoma Yelingue *, Wenddabo Olivier Sawadogo, Blaise Some \\ Laboratoire d'Analyse Numerique d'Informatique et de BIOmathematique Universite de Ouaga 1, \\ Joseph KI Zerbo, 03 BP 7021 Ouagadougou 03, Burkina Faso \\ *Corresponding author E-mail: bensthal@gmail.com
}

\begin{abstract}
In this paper the partial derivative equation strongly nonlinear of Richards which models the dynamics of water in the Un-saturated Zone (UZ) was linearized and solved by a new numerical method called SBA. The analytical solution has been simulated in order to be applied later to the following in unsaturated zone with the aquifers of Bangui and its boundaries.
\end{abstract}

Keywords: Analytical Solution; Linearization; Richards' Equation; SBA Method; Unsaturated Zone (UZ).

\section{Introduction}

In this paper we consider the following Richards' equation [5], [7]:

$$
\left\{\begin{array}{ccc}
\frac{\partial \theta}{\partial t}=\vec{\nabla}[K(h) \vec{\nabla}(h-z)] & , \quad(z, t) \in \Omega \times[0, T] \\
h(z, 0)=h_{C I}(z) & , & z \in \delta \Omega \\
h(z, t)=h_{C L}(z, t) &
\end{array}\right.
$$

Where

$K\left[\boldsymbol{L} \cdot T^{-1}\right]$ : Is the soil hydraulic conductivity at the point considered which represents the 2 nd tensor order in general case, reduced to a scalar where the soil is isotropic ; $H[L]=h-z$ is the hydraulic charge at the considered point; $\mathrm{h}[\mathrm{L}]$ as water pressure relatively to atmospheric pressure and expressed in terms of water height; $z[L]$ the side of considered point positively measured downwards with ground surface as reference point; $\operatorname{grad}(H)$ is the gradient operator; $\theta\left[\mathrm{m}^{3} / \mathrm{m}^{3}\right]$ volumetric water content The diffuse form of Richards' equation that introduces soil diffusivity $D(\theta)$ expressed in $\left[L^{2} T^{-1}\right]$ and which privileges resolution in $\theta$ and the capacitive form of Richards' equation which introduces the soil capillary capacity $C(h)$ expressed by $\left[L^{-1}\right]$ and which privileges the resolution in $h$.

For a vertical flow in space dimension 1 , continuity equation in $\theta$ is known as Fokker-Planck equation:

$$
\frac{\partial \theta}{\partial t}=\frac{\partial}{\partial z}\left(D(\theta) \cdot \frac{\partial \theta}{\partial z}\right)+\frac{\partial K(\theta)}{\partial z}
$$

And in $\mathrm{h}$ the equation is known under the name of Richards equation [1931]:

$$
C(h) \frac{\partial h}{\partial t}=\frac{\partial}{\partial z}\left(K(h)\left(\frac{\partial h}{\partial z}-1\right)\right)
$$

In the following, the form (3) will be used:

We can define the Richards model to solve in dimension 1 of space in $\mathrm{z}$ :

$$
\left\{\begin{array}{ccc}
C(h) \frac{\partial h}{\partial t}=\frac{\partial}{\partial z}\left[K(h)\left(\frac{\partial h}{\partial z}-1\right)\right] & , & (z, t) \in \Omega \times[0, T] \\
h(z, 0)=h_{C I}(z) & , \quad z \in \delta \Omega \\
h(z, t)=h_{C L}(z, t) &
\end{array}\right.
$$

Two functions inducing the nonlinearities that are hydraulic Conductivity K (h) and the Capillary Capacity C (h) [2].

These functions depend on the variation of the water content $\theta$ and the matrix pressure $\mathrm{h}$. It is a strongly nonlinear parabolic PDE whose existence and unicity of the solution are proven in $[8,4]$. SBA Method [14] has been used to determine the analytical solution after linearization of the functions $\mathrm{K}(\mathrm{h})$ and $\mathrm{C}(\mathrm{h})$. Many digital method does not converge because of the strong nonlinearity if we want to solve the Richards equation. So it uses the SBA method to the advantage didn't discredited and maintains the physical properties of the model parameters and converges despite the nonlinearity.

\section{Theoretical description of method SBA}

Method S.B.A. (SOME Blaise ABBO) allows to solve functional equations of type: ODE, system of ODE, PDE, system of nonlinear and strongly nonlinear PDE under certain conditions. It also applies to linear problems. This new algorithm makes it possible to obtain exact solutions of some ODE, system of ODE, PDE, system of nonlinear and strongly nonlinear PDE with initial conditions or initial conditions and in extreme cases. The new technique brings back the resolution of any problem of PDE (resp. ODE) nonlinear with initial conditions and in extreme cases to the solution of an equation of the 
Cauchy type. Based on a combination of the décompositionnelle method of Adomian an idea of the method of the successive approximations and the method of Picard this algorithm is with fast convergence (one or two iterations) towards the exact solution of the PDE (resp. ODE), system of PDE (resp. system of PDE) if they exist.

The details, the convergence and the mathematical framework of algorithm SBA are milked in [3], 9], [11], [13].

\subsection{Principles of the method}

On the basis of a problem ODE, system of ODE, PDE, system of linear and strongly nonlinear EDP, method SBA (SOME Blaise Abbo) consists with:

- To approach the initial problem by the iterative diagram by using the idea of the method of the successive approximations and this, in suitable functional spaces. The resolution of the iterative diagram by the same method amounts determining with each iteration $(\mathrm{k}=1,2,3 \ldots$ of the approximate solutions which are obtained by using the method of Picard and this after a judicious choice of the initial $U^{0}$ and the solution of the problem is obtained by taking the limit $\left(U^{k}\right)_{k}$ of the continuation

- To reconsider the iterative diagram by using the method of the successive approximations either but rather décompositionnelle method of Adomian to each stage of iteration, under the judicious choice of $U^{0}$.

- To deduce the algorithm from Adomian on the basis of a canonical form of Adomian.

- To obtain the solution with each stage then the general solution by a calculation of limit.

\subsection{Solving Richards equation by SBA}

\subsubsection{Linearization functions $C(h)$ and $K(h)$}

This linearization will be based on two concepts including the concept of sucking and the notion of Limited Developments (L.D.) to order $\mathrm{n}$ with application to the case $\mathrm{n}=5$. The function $\mathrm{C}(\mathrm{h})$ and $\mathrm{K}$ (h) respectively represent the capillary capacity and hydraulic conductivity. They are written as a function of the pressure charge (charge matrix) h. The used forms in our case are taken from [2]. They induce nonlinearities in equation (4), complicating its resolution with lot of methods. They are empirically defined [2]:

$$
C(h)=\frac{\theta_{s}(2-n)}{h_{g}}\left(\frac{h}{h_{g}}\right)^{n-1}\left[1+\left(\frac{h}{h_{g}}\right)^{n}\right]^{\frac{2}{n-2}}
$$

, if $h \pi 0$ unsaturated case

\subsubsection{Sucking concept}

Generally, in unsaturated area, the pressure charge (load matrix) h is always negative; it is frequently replaced by the suction $\Psi$ defined by [9]:

$$
\Psi=|h|
$$

Where $\Psi$ can be expressed as $P a$ or $b a r$.

\subsubsection{Limited development concept order $\mathbf{n}$ in 0}

In the situation thereafter, we will be interested only in the following forms:

$$
e^{y}=g(y)=\sum_{i=0}^{n}\left(\frac{y^{i}}{i !}\right)+R_{1}
$$

Whereby, $R_{1}$ is the rest of the L.D.?

$$
\ln (1+v)=h(v)=\sum_{j=1}^{n}(-1)^{j+1}\left(\frac{v^{. j}}{j}\right)+R_{2}
$$

Where, $R_{1}$ is the rest of the L.D.?

Based on these two concepts and proceeding in (5) and (6) the following variables changes according to where we must then express functions $C(h)$ and $K(h)$ in function of the variable $\Psi$ then the variable $S$, we will get successively:

In (5), expression of $C=f(\Psi)$ :

We set successively

$$
\begin{array}{r}
U=\frac{h}{h_{g}}, \quad h=h_{g} U, \quad|h|=h_{g}|U|, \\
|U|=\frac{\Psi}{h_{g}}=S, \quad \text { and } \quad c=\frac{\theta_{s}(2-n)}{h}
\end{array}
$$

(5) Becomes

$$
C(U)=c U^{n-1}\left[1+U^{n}\right]^{\frac{2}{n}-2}
$$

Moreover

$$
C(|\boldsymbol{U}|)=c|\boldsymbol{U}|^{n-1}\left[1+|\boldsymbol{U}|^{n}\right]^{\frac{2}{n}-2}
$$

As Then (13) becomes:

$$
C(\Psi)=c\left(\frac{\Psi}{h_{g}}\right)^{n-1}\left[1+\left(\frac{\Psi}{h_{g}}\right)^{n}\right]^{\frac{2}{n}-2}
$$

And (14) becomes

$$
C(S)=c S^{n-1}\left[1+S^{n}\right]^{\frac{2}{n}-2}
$$

(15) Can be rewriten by:

$$
C(S)=c e^{(n-1) \ln (S)} e^{\left(\frac{2}{n}-2\right) \ln \left(1+S^{n}\right)}
$$

In the following, we will proceed to the LD order 5 around 0 of (16).

- $\quad$ LD order 5 of (16) around 0.

By setting successively:

$$
r=(n-1) \ln (S), V=S^{n}, b=\frac{2}{n}-2, Y=b \ln (1+V)
$$


$q=1-\frac{1}{2}\left(S^{n}\right)+\frac{1}{3}\left(S^{n}\right)^{2}-\frac{1}{4}\left(S^{n}\right)^{3}+\frac{1}{5}\left(S^{n}\right)^{4}$

And $p=b q\left(S^{n}\right)$ we get:

$e^{(n-1) \ln (S)}=e^{r}=\sum_{i=0}^{5}\left(\frac{r^{i}}{i !}\right)+R_{3}$

$\ln (1+V)=\sum_{k=0}^{5}(-1)^{k+1}\left(\frac{V^{k}}{k}\right)+R_{4}=\ln \left(1+S^{n}\right)$

$\ln \left(1+S^{n}\right)=\sum_{j=0}^{5}(-1)^{j+1} \frac{\left(S^{n}\right)^{i}}{i}+R_{5}$

$e^{b \ln \left(1+S^{n}\right)}=e^{Y}=\sum_{p=0}^{5}\left(\frac{Y^{p}}{p !}\right)+R_{6}$

Where $R_{3}, R_{4}, R_{5}, R_{6}$ are the rest of the LD.

According to (19), (20) becomes:

$e^{b \ln \left(1+S^{n}\right)}=\sum_{i=0}^{5} b^{i}\left(S^{n}\right)^{i} q^{i}+R_{7}$

Where $R_{7}$ is the rest of the DL?

(16) Becomes:

$C(S)=c\left[\sum_{i=0}^{5}\left(\frac{r^{i}}{i !}\right)+R_{3}\right]\left[\sum_{j=0}^{5}\left(\frac{p^{j}}{j !}\right)+R_{4}\right]$

That mens

$C(S)=c \Phi(r) \Gamma(p)$

With

$\Phi(r)=e^{r}=\sum_{i=0}^{5}\left(\frac{r^{i}}{i !}\right)+R_{3}$

$\Gamma(p)=e^{p}=\sum_{j=0}^{5}\left(\frac{p^{j}}{j !}\right)+R_{4}$

At this level, the result consists of comparing the curve of (16) with the curve of (23), so that (23) has been substituted from Richards equation.

- In (6), expression of $K=f(\Psi)$

We set

$$
\begin{gathered}
U=\frac{h}{h_{g}}, \quad h=h_{g} U, \quad|h|=h_{g}|U|, \\
|U|=\frac{\Psi}{h_{g}}=S, \quad \text { and } \quad w=m\left(\frac{2}{n}-1\right)
\end{gathered}
$$

(6) Becomes:
$K(U)=K_{s}\left[1+U^{n}\right]^{w}$

(27) Becomes successively:

$$
\begin{aligned}
& K(|U|)=K_{s}\left[1+|U|^{n}\right]^{w} \\
& K(\Psi)=K_{s}\left[1+\left(\frac{\Psi}{h_{g}}\right)^{n}\right]^{w}
\end{aligned}
$$

And (29) becomes:

$$
K(S)=K_{s}\left[1+(S)^{n}\right]^{w}
$$

(30) Can be written:

$$
K(S)=K_{s} e^{w \ln \left(1+S^{n}\right)}
$$

Similarly, we will proceed thereafter, to LD order 5 of (31) Around 0.

By setting successively:

$X=S^{n}$ et $Y=w \ln (1+X)$

$K(Y)=K_{S} e^{Y}$

$\ln (1+X)=\sum_{i=1}^{5}(-1)^{i+1} \frac{(X)^{i}}{i}+R_{5}$

$e^{Y}=\sum_{j=0}^{5} \frac{Y^{j}}{j !}+R_{6}$

With

$Y=\left[w\left(S^{n}\right)-\frac{w}{2}\left(S^{n}\right)^{2}+\frac{w}{3}\left(S^{n}\right)^{3}-\frac{w}{4}\left(S^{n}\right)^{4}+\frac{w}{5}\left(S^{n}\right)^{5}\right]+R_{7}$

Which upon reduction gives:

$K(S)=K_{s} e^{y}=\left[\begin{array}{l}1+w\left(S^{n}\right)-\left(\frac{w}{3}-\frac{w^{2}}{2}+\frac{w^{3}}{6}\right)\left(S^{n}\right)^{3}+\left(\frac{w}{4}+\frac{5 w^{2}}{24}+\frac{w^{4}}{24}\right)\left(S^{n}\right)^{4}+ \\ +\left(\frac{w}{5}-\frac{w^{2}}{4}+\frac{5 w^{3}}{24}-\frac{w^{4}}{24}\right)\left(S^{n}\right)^{5}\end{array}\right]$

Which can be rewritten:

$K(S)=K_{S}\left[1+\alpha\left(S^{n}\right)-\beta\left(S^{n}\right)^{3}+\gamma\left(S^{n}\right)^{4}+\lambda\left(S^{n}\right)^{5}\right]=K_{s}+K_{S} w\left(S^{n}\right)+\varphi(S)$

With

$\varphi(S)=-\beta\left(S^{n}\right)^{3}+\gamma\left(S^{n}\right)^{4}+\lambda(S)^{5}$

With

$\alpha=w, \beta=\frac{w}{3}-\frac{w^{2}}{2}+\frac{w^{3}}{6}$,

$\gamma=-\frac{w}{4}+\frac{5 w^{2}}{24}-\frac{w^{3}}{4}+\frac{w^{4}}{24}$ 


$$
\lambda=\frac{w}{5}-\frac{w^{2}}{4}+\frac{5 w^{3}}{24}-\frac{w^{4}}{24}
$$

In the next situation, the curves of (30) and (37) have been compared in order to substitute (37) in the PDE of Richards.

\subsubsection{Conclusion}

Through this linearization, we were able to determine appropriate forms of functions $\mathrm{C}(\mathrm{h})$ and $\mathrm{K}(\mathrm{h})$ that will allow us to substitute them from equation called Richards one to get the modified form that obeys utilization conditions of SBA method.

Note: We also note that the LD order 5 used so far can be extended to the order $\mathrm{n}$, and the result will remain the same

\subsubsection{Richards' equation amended to 1D}

Let's consider Richards model in 1D in $\mathrm{z}$ expressed in terms of charge pressure $\mathrm{h}$ defined in (4):

$$
\left\{\begin{array}{ccc}
C(h) \frac{\partial h}{\partial t}=\frac{\partial}{\partial z}\left[K(h)\left(\frac{\partial h}{\partial z}-1\right)\right] & , & (z, t) \in \Omega \times[0, T] \\
h(z, 0)=h_{C I}(z) & , & z \in \delta \Omega \\
h(z, t)=h_{C L}(z, t) &
\end{array}\right.
$$

To determine then the shape of the amended equation, we will use the linearized forms of C (h) and $\mathrm{K}(\mathrm{h})$ established in (34) and (48).

- Let's express the model (51) called Richards in 1D according to the suction $\Psi=|h|$.

In this case (40) becomes:

$$
\left\{\begin{array}{ccc}
C(|h|) \frac{\partial(|h|)}{\partial t}=\frac{\partial}{\partial z}\left[K(|h|)\left(\frac{\partial(|h|)}{\partial z}-1\right)\right] & , & (t, z) \in \Omega \times[0, T] \\
|h(0, z)|=\left|h_{C I}(z)\right| & , \quad(t, z) \in \delta \Omega \times[0, T] \\
|h(t, z)|=\left|h_{C L}(t, z)\right| &
\end{array}\right.
$$

From the foregoing,

$$
\left\{\begin{array}{cc}
C(\Psi) \frac{\partial(\Psi)}{\partial t}=\frac{\partial}{\partial z}\left[K(\Psi)\left(\frac{\partial(\Psi)}{\partial z}-1\right)\right] & , \quad(t, z) \in \Omega \times[0, T] \\
\Psi(t, z)=\Psi_{C I}(z) & , \quad(t, z) \in \delta \Omega \times[0, T] \\
\Psi(t, z)=\Psi_{C L}(t, z) & , \quad(t, z) \in \Omega \times[0, T]
\end{array}\right.
$$

Therefore the equation (53) can be rewritten as follows:

$$
\left\{\begin{array}{ccc}
C(\Psi) \frac{\partial(\Psi)}{\partial t}=K(\Psi) \frac{\partial^{2} \Psi}{\partial z^{2}}+\frac{\partial K(\Psi)}{\partial z} \frac{\partial \Psi}{\partial z}-\frac{\partial K(\Psi)}{\partial z} & , & (t, z) \in \Omega \times[0, T] \\
\Psi(t, z)=\Psi_{C I}(z) & , & (t, z) \in \delta \Omega \times[0, T] \\
\Psi(t, z)=\Psi_{C L}(t, z) & , & (t, z) \in \Omega \times[0, T]
\end{array}\right.
$$

- Let's express (30) and (37) as a function of $\Psi$ We get (30): $C(S)=c \Phi(r) \Gamma(p)=c\left[\sum_{i=0}^{5}\left(\frac{r^{i}}{i !}\right)+R_{3}\right]\left[\sum_{j=0}^{5}\left(\frac{p^{j}}{j !}\right)+R_{4}\right]=c .1 .\left[\sum_{j=0}^{5}\left(\frac{p^{k}}{k !}\right)+R_{4}\right]+c \cdot h(r, p)$

That means

$$
C(S)=c \Phi(r) \Gamma(p)=c .1 .\left[1+p+\frac{1}{2} p^{2}+\frac{1}{6} p^{3}+\frac{1}{24} p^{4}+\frac{1}{120} p^{5}\right]+c \cdot h(r, p)
$$

With

$$
h(r, p)=\left[\sum_{i=1}^{5}\left(\frac{r^{i}}{i !}\right)+R_{3}\right]\left[\sum_{k=0}^{5}\left(\frac{p^{k}}{k !}\right)+R_{4}\right]
$$

However $h(r, p)=f(S)$ because $\Gamma(p)=f(S)$ et $\Phi(r)=f(S)$ and

$h(r, p)=f(S)$

Then we can write that

$. \Phi(r) . \Gamma(p)=[1+g(p)+h(r, p)]=1+g(S)+h(S)$

With

$$
g(p)=\sum_{k=1}^{5}\left(\frac{p^{k}}{k !}\right)+R_{4} \text { And } h(S)=h(r, p)
$$

Then the function $C(S)$ can be rewritten:

$$
C(S)=c[1+g(S)+h(S)]=c+c \cdot g(S)+c \cdot h(S)
$$

Then

$$
C(S)=c+c[g(S)+h(S)]
$$

Very important note:

If we switch the function $\Phi(r) \Gamma(p)$ by $\Gamma(p) \Phi(r)$ another less interesting form will be found out of $C(S)$ that cannot take into account all parameters of the studied model.

- Let's express (43) according to

$$
S=\frac{\Psi}{h_{g}}
$$

Therefore (43) becomes:

$$
\left\{\begin{array}{cc}
c\left(h_{g} S\right) \frac{\partial\left(h_{g} S\right)}{\partial t}=K\left(h_{g} S\right) \frac{\partial^{2}\left(h_{g} S\right)}{\partial Z^{2}}+\frac{\partial K\left(h_{g} S\right)}{\partial z} \frac{\partial\left(h_{g} S\right)}{\partial z}-\frac{\partial\left(h_{g} S\right)}{\partial z} & , \quad(t, z) \in[0, T] \times \Omega \\
S(0, z)=S_{C l}(z) & ,(t, z) \in[0, T] \times \delta \Omega \\
S(t, z)=S_{C L}(t, z) & , \quad(t, z) \in[0, T] \times \Omega
\end{array}\right.
$$

By clarifying the boundary and initials conditions, (50) system after reduction is equivalent to the following system:

$$
\left\{\begin{array}{c}
C(S) \frac{\partial S}{\partial t}=\frac{\partial K(S)}{\partial Z} \frac{\partial S}{\partial Z}+K(S) \frac{\partial^{2} S}{\partial Z^{2}}-\frac{1}{h_{g}} \frac{\partial K(S)}{\partial Z} \\
S(0, Z)=S_{\text {init }}(Z) \\
S(t, 0)=S_{\text {surf }}(t) \\
S(t, Z)=S_{\text {fond }}(t)
\end{array}\right.
$$

- Let's break $C(S) \frac{\partial S}{\partial t}$

Out of (49) by taking into account (51), there are obtained successively:

$C(S) \frac{\partial S}{\partial t}=[c+c(g(S)+h(S))] \frac{\partial S}{\partial t}=c \frac{\partial S}{\partial t}+c g(S) \frac{\partial S}{\partial t}+c h(S) \frac{\partial S}{\partial t}$ 
- Similarly decomposing $\frac{\partial K(S)}{\partial z} \frac{\partial S}{\partial z}, K(S) \frac{\partial^{2} S}{\partial z^{2}}$ and $\frac{\partial K(S)}{\partial z}$

$\partial z$ out of (51) and taking into account (38), there have resulted successively:

$$
\begin{aligned}
& \frac{\partial K(S)}{\partial z} \frac{\partial S}{\partial z}=\frac{\partial}{\partial z}\left[K_{S}+K_{S} w\left(S^{n}\right)^{4}+\varphi(S)\right] \frac{\partial S}{\partial z}= \\
& =\frac{\partial K_{S}}{\partial z} \frac{\partial S}{\partial z}+K_{S} w \frac{\partial\left(S^{n}\right)}{\partial z} \frac{\partial S}{\partial z}+\frac{\partial \varphi(S)}{\partial z} \frac{\partial \varphi(S)}{\partial z} \frac{\partial S}{\partial z}
\end{aligned}
$$

As

$K_{S}=C t e \Rightarrow \frac{\partial K_{S}}{\partial z}=0$

then

$$
\begin{aligned}
& \frac{\partial K(S)}{\partial z} \frac{\partial S}{\partial z}=K_{S} w \frac{\partial\left(S^{n}\right)}{\partial z} \frac{\partial S}{\partial z}+\frac{\partial\left(S^{n}\right)}{\partial z} \frac{\partial S}{\partial z} \\
& K(S) \frac{\partial^{2} S}{\partial Z^{2}}=\left[K_{s}+K_{s} w S^{n}+\varphi(S)\right] \frac{\partial^{2} S}{\partial Z^{2}} \\
& K(S) \frac{\partial^{2} S}{\partial Z^{2}}=K_{s} \frac{\partial^{2} S}{\partial Z^{2}}+\left[K_{s} w S^{n}+\varphi(S)\right] \frac{\partial^{2} S}{\partial Z^{2}} \\
& \frac{\partial K(S)}{\partial Z}=\frac{\partial\left[K_{s}+K_{s} w S^{n}+\varphi(S)\right]}{\partial Z}=\frac{\partial K_{S}}{\partial Z}+K_{s} w \frac{\partial S^{n}}{\partial Z}+\frac{\partial \varphi(S)}{\partial Z}
\end{aligned}
$$

As

$$
K_{S}=C t e
$$

Then

$$
\begin{aligned}
& \frac{\partial K_{s}}{\partial Z}=0 \\
& \frac{\partial K(S)}{\partial Z}=K_{s} w \frac{\partial S^{n}}{\partial Z}+\frac{\partial \varphi(S)}{\partial Z}
\end{aligned}
$$

So by combining the relations (52), (54), (56) and (58), (51) becomes:

$$
\left\{\begin{aligned}
c \frac{\partial S}{\partial t}+c g(S) \frac{\partial S}{\partial t}+c h(S) \frac{\partial S}{\partial t}=K_{s} w \frac{\partial S^{n}}{\partial Z} \frac{\partial S}{\partial Z}+\frac{\partial \varphi(S)}{\partial Z} \frac{\partial S}{\partial Z}+K_{s} \frac{\partial^{2} S}{\partial Z^{2}}+ \\
+\left[K_{s} w S^{n}+\varphi(S)\right] \frac{\partial^{2} S}{\partial Z^{2}}-\frac{K_{s} w}{h_{g}} \frac{\partial S^{n}}{\partial Z}-\frac{1}{h_{g}} \frac{\partial \varphi(S)}{\partial Z} \\
S(0, Z)=S_{\text {init }}(Z) \\
S(t, 0)=S_{\text {surf }}(t) \\
S(t, Z)=S_{\text {fond }}(t)
\end{aligned}\right.
$$

(59) Is equivalent to the following?

$$
\left\{\begin{aligned}
c \frac{\partial S}{\partial t}=K_{s} \frac{\partial^{2} S}{\partial Z^{2}}-\left(\left[c g(S) \frac{\partial S}{\partial t}+c h(S) \frac{\partial S}{\partial t}\right]+\left[K_{s} w \frac{\partial S^{n}}{\partial Z} \frac{\partial S}{\partial Z}+\frac{\partial \varphi(S)}{\partial Z} \frac{\partial S}{\partial Z}\right]+\right. \\
\left.+\left[\left[K_{s} w S^{n}+\varphi(S)\right] \frac{\partial^{2} S}{\partial Z^{2}}\right]-\left[\frac{K_{s} w}{h_{g}} \frac{\partial S^{n}}{\partial Z}+\frac{1}{h_{g}} \frac{\partial \varphi(S)}{\partial Z}\right]\right) \\
S(0, Z)=S_{\text {init }}(Z) \\
S(t, 0)=S_{\text {surf }}(t) \\
S(t, Z)=S_{\text {fond }}(t)
\end{aligned}\right.
$$

Whereby

$$
c=\frac{\theta_{s}(2-n)}{h_{g}}
$$

Relation (60) can still be rewritten after reduction to give ultimately qualified model of the Richards Equation Modified in 1D in $\mathrm{z}$, we will resolve by SBA method:

$$
\left\{\begin{array}{c}
\frac{\partial S}{\partial t}=\frac{K_{s}}{c} \frac{\partial^{2} S}{\partial Z^{2}}-\left(\left[g(S) \frac{\partial S}{\partial t}+h(S) \frac{\partial S}{\partial t}\right]+\left[\frac{K_{s} w}{c} \frac{\partial S^{n}}{\partial Z} \frac{\partial S}{\partial Z}+\frac{1}{c} \frac{\partial \varphi(S)}{\partial Z} \frac{\partial S}{\partial Z}\right]+\right. \\
\left.+\frac{1}{c}\left[\left[K_{s} w S^{n}+\varphi(S)\right] \frac{\partial^{2} S}{\partial Z^{2}}\right]-\left[\frac{K_{s} w}{h_{g} c} \frac{\partial S^{n}}{\partial Z}+\frac{1}{h_{g} c} \frac{\partial \varphi(S)}{\partial Z}\right]\right) \\
S(0, Z)=S_{\text {init }}(Z)=\theta(Z) \\
S(t, 0)=S_{\text {suff }}(t)=\alpha(t) \\
S(t, Z)=S_{\text {fond }}(t)=\beta(t)
\end{array}\right.
$$

We noticed that all scale parameters in the Hydraulic Conductivity $K(h)$ and Capillary Capacity $C(h)$ are taken into account in the system (61) and its parameters are : $K_{s}, \theta_{s}$ and $h_{g}$ as well as the shape parameters such as $\mathrm{m}$ and $\mathrm{n}$.

The system (61) is the new model of modified Richards, which is also a highly nonlinear PDEs but easily solvable with the SBA method with initial and limit conditions.

The system (61) can be put in the following form, which form will allow us to apply the SBA algorithm if we set for example the following conditions:

$$
\left\{\begin{array}{c}
S_{t}=\frac{K_{s}}{c} S_{Z Z}+N(S(t, Z)), \mathrm{K} a \leq Z \leq b, \mathrm{~K} t \geq 0 \\
\cdot \\
S(0, Z)=\theta(Z) \\
S(t, 0)=\alpha(t) \\
S(t, Z)=\beta(t)
\end{array}\right.
$$

Or

$$
\begin{aligned}
& N(S(t, Z))=-\left[g(S) \frac{\partial S}{\partial t}+h(S) \frac{\partial S}{\partial t}\right]+ \\
& +\left[\frac{K_{s} w}{c} \frac{\partial S^{n}}{\partial Z} \frac{\partial S}{\partial Z}+\frac{1}{c} \frac{\partial \varphi(S)}{\partial Z} \frac{\partial S}{\partial Z}+\frac{1}{c}\left(K_{s} w S^{n}-\varphi(S)\right) \frac{\partial^{2} S}{\partial Z^{2}}\right]- \\
& -\left[\frac{K_{s} w}{c h_{g}} \frac{\partial S^{n}}{\partial Z}+\frac{1}{h_{g} c} \frac{\partial \varphi(S)}{\partial Z}\right]
\end{aligned}
$$

Then we have been able to set Richards model as follows:

$$
\left\{\begin{array}{c}
S_{t}=L(S(t, Z))+N(S(t, Z)), \mathrm{K} a \leq Z \leq b, \mathrm{~K} t \geq 0 \\
\cdot \\
S(0, Z)=\theta(Z) \\
S(t, 0)=\alpha(t) \\
S(t, Z)=\beta(t)
\end{array}\right.
$$

Whereby ${ }^{L}$ and ${ }^{N}$ are respectively linear and nonlinear operators such as:

$$
L(S)=L_{1}(S)+L_{2}\left(S_{Z}, S_{Z Z}, S_{Z Z Z}, \ldots\right)_{\text {Avec }} L_{2} \neq \mathbf{O}
$$

Very important Remark:

In the Richards model used until then, it is assumed that the nonlinear PDE subject to SBA algorithm is homogeneous every time we 
can still consider the non-homogeneous case where there is presence of sources or sinks $\phi$ that is to say:

- $L(U)+N(U)=0$

$L(U)+N(U)=\phi$

We will in the following resolve theoretically the model (64) by applying S.BA method.

\subsubsection{Application of SBA method}

\subsubsection{Theoretical application of SBA}

The considered system is (64):

$$
\left\{\begin{array}{c}
S_{t}=\frac{K_{s}}{c} S_{Z Z}+N(S(t, Z)), \mathrm{K} a \leq Z \leq b, \mathrm{~K} t \geq 0 \\
\cdot \\
S(0, Z)=\theta(Z) \\
S(t, 0)=\alpha(t) \\
S(t, Z)=\beta(t)
\end{array}\right.
$$

Or

$$
\begin{aligned}
& N(S(t, Z))=-\left[g(S) \frac{\partial S}{\partial t}+h(S) \frac{\partial S}{\partial t}\right]+ \\
& +\left[\frac{K_{s} w}{c} \frac{\partial S^{n}}{\partial Z} \frac{\partial S}{\partial Z}+\frac{1}{c} \frac{\partial \varphi(S)}{\partial Z} \frac{\partial S}{\partial Z}+\frac{1}{c}\left(K_{s} w S^{n}-\varphi(S)\right) \frac{\partial^{2} S}{\partial Z^{2}}\right]- \\
& -\left[\frac{K_{s} w}{c h_{g}} \frac{\partial S^{n}}{\partial Z}+\frac{1}{h_{g} c} \frac{\partial \varphi(S)}{\partial Z}\right]
\end{aligned}
$$

In the following, we ask $\alpha(t)=\alpha$ and $\beta(t)=\beta$.

So the final problem to be solved by this algorithm is:

$$
\left\{\begin{array}{c}
S_{t}=\frac{K_{s}}{c} S_{\mathrm{ZZ}}+N(S(t, Z)), \mathrm{K} a \leq Z \leq b, \mathrm{~K} t \geq 0 \\
\cdot \\
S(0, Z)=\theta(Z) \\
S(t, 0)=\alpha \\
S(t, Z)=\beta
\end{array}\right.
$$

Whereby, $N(S(t, Z))$ is defined as previously.

Problem of the transformation (67) into a Cauchy type of problem: 1) Taking account of edge conditions:

Consider the following equation:

$$
S_{t}=\frac{K_{s}}{c} S_{Z Z}+N(S(t, Z))
$$

Let's set $\frac{K_{s}}{c}=\lambda$.

Then the above equation can be written:

$$
S_{t}-\lambda S_{z Z}-N(S)=0 \text {. }
$$

Consider the following operators:

$$
L_{t}=\frac{\partial()}{\partial t} \Rightarrow L_{t}^{-1}=\int_{0}^{t}(\bullet) d u
$$

$L_{\mathrm{ZZ}}^{2}=\frac{\partial^{2}(\bullet)}{\partial Z^{2}} \Rightarrow L_{\mathrm{ZZ}}^{-2}=\int_{a}^{b} \int_{a}^{b}(\bullet) d r d r$

a) Consider firstly the operator $L_{t}$ of reverse $L_{t}^{-1}$ the egality (a) becomes :

$L_{t} S-\lambda L_{Z Z} S-N(S)=0$

By applying the reverse $L_{t}^{-1}$ we get:

$L_{t}^{-1} L_{t}-\lambda L_{t}^{-1} L_{Z Z} S-L_{t}^{-1} N(S)=0$

This gives the following relationship:

$$
\int_{0}^{t} \frac{\partial S(u, Z)}{\partial u} d u-\lambda \int_{0}^{t} \frac{\partial^{2} S(u, Z)}{\partial Z^{2}} d u-\int_{0}^{t} N(S(u, Z)) d u=0
$$

$[S(u, Z)]_{0}^{t}-\lambda \int_{0}^{t} \frac{\partial^{2} S(u, Z)}{\partial Z^{2}} d u-\int_{0}^{t} N(S(u, Z)) d u=0$

$S(t, Z)-S(0, Z)-\lambda \int_{0}^{t} \frac{\partial^{2} S(u, Z)}{\partial Z^{2}} d u-\int_{0}^{t} N(S(u, Z) d u)=0$

Whereby we have:

$S(t, Z)=S(0, Z)+\lambda \int_{0}^{t} \frac{\partial^{2} S(u, Z)}{\partial Z^{2}} d u-\int_{0}^{t} N(S(u, Z)) d u$

b) Let's consider the other operator

$\boldsymbol{L}_{Z Z}^{2}$ Reverse $\boldsymbol{L}_{Z Z}^{-2}$ equality (a) becomes:

$L_{t} s-\lambda L_{Z Z}^{2} s-\lambda(S)=\mathbf{O}$

By applying the reverse $\boldsymbol{L}_{Z Z}^{-2}$ of $\boldsymbol{L}_{Z Z}^{2}$ we have:

$L_{Z Z}^{-2} L_{t} S-\lambda L_{Z Z}^{-2} L_{Z Z}^{2} S-L_{Z Z}^{-2} N(S)=0$

We have the following relationship:

$\int_{a}^{b} \int_{a}^{b} \frac{\partial S(t, V)}{\partial t} d V d V-\lambda \int_{a}^{b} \int_{a}^{b} \frac{\partial^{2} S(t, V)}{\partial V^{2}} d V d V-\int_{a}^{b} \int_{a}^{b} N(S(t, V)) d V d V=0$,

Which gives:

$\lambda \int_{a}^{b} \int_{a}^{b} \frac{\partial^{2} S(t, V)}{\partial V^{2}} d V d V=+\int_{a}^{b} \int_{a}^{b} \frac{\partial S(t, V)}{\partial t} d V d V-\int_{a}^{b} \int_{a}^{b} N(S(t, V)) d V d V$,

Which gives:

$\int_{a}^{b} \int_{a}^{b} \frac{\partial^{2} S(t, V)}{\partial V^{2}} d V d V=\frac{1}{\lambda} \int_{a}^{b} \int_{a}^{b} \frac{\partial S(t, V)}{\partial t} d V d V-\frac{1}{\lambda} \int_{a}^{b} \int_{a}^{b} N(S(t, V)) d V d V$,

Which gives:

$$
S(a, t)-S(b, t)-\frac{1}{\lambda} \int_{a}^{b} \int_{a}^{b} \frac{\partial S(t, V)}{\partial t} d V d V+\frac{1}{\lambda} \int_{a}^{b} \int_{a}^{b} N(S(t, V)) d V d V=0
$$

By combining the equation (1) and (2) we have: 


$$
\begin{aligned}
& S(t, Z)=S(0, Z)-\lambda \int_{0}^{t} \frac{\partial^{2} S(u, Z)}{\partial Z^{2}} d u+\int_{0}^{t} N(S(u, Z)) d u+S(a, t)-S(b, t)- \\
& -\frac{1}{\lambda} \int_{a}^{b} \int_{a}^{b} \frac{\partial S(t, V)}{\partial t} d V d V+\frac{1}{\lambda} \int_{a}^{b} \int_{a}^{b} N(S(t, V) d V d V)
\end{aligned}
$$

We obtain the following Cauchy problem type:

$$
\left\{\begin{array}{c}
S(t, Z)=S(a, t)-S(b, t)+S(0, Z)-\lambda \int_{0}^{t} \frac{\partial^{2} S(u, Z)}{\partial Z^{2}}+\int_{0}^{t} N(S(u, Z)) d u- \\
-\frac{1}{\lambda} \int_{a}^{b} \int_{a}^{b} \frac{\partial S(t, V)}{\partial t} d V d V+\frac{1}{\lambda} \int_{a}^{b} \int_{a}^{b} N(S(t, V)) d V d V \\
S(0, Z)=\theta(Z)
\end{array}\right.
$$

The relation (68) is called canonical form of Adomian.

The approximate equation of the problem (68) by the new Adomian technique can be written:

$$
\left\{\begin{array}{c}
644444444^{L t y^{k}} 444444448 \\
S^{k}(t, Z)=S^{k}(a, t)-S^{k}(b, t)+S^{k}(0, Z)-\lambda \int_{0}^{t} \frac{\partial^{2} S^{k}(u, Z)}{\partial Z^{2}}+ \\
+\int_{0}^{t} N\left(S^{k-1}(u, Z)\right) d u-\frac{1}{\lambda} \int^{b} \int_{4^{a}}^{b} \frac{\partial S^{k-1}(t, V)}{\partial t 424} d V d V+\frac{1}{\lambda} \int^{b} \int_{4^{a}}^{b} N\left(S^{k-1}(t, V)\right) d V d V \\
\left.9444444444 u^{k-1}\right) \\
S^{k}(0, Z)=\theta(Z), \mathrm{K} k=1,2, \mathrm{~K} \\
S^{k}(t, 0)=\alpha(t), \mathrm{K} k=1,2, \mathrm{~K} \\
S^{k}(t, Z)=\beta(t), \mathrm{K} k=1,2, \mathrm{~K}
\end{array}\right.
$$

Wherby

$$
S^{k}(t, Z)=L\left(u^{k}\right)+N\left(u^{k-1}\right)
$$

This canonical form obtained by the new approach Adomian allows, following the technique developed to resolve the problem (69).

Indeed, the resolution of the above pattern (S.B.A.) by the method of successive approximations, consists then in determining for each iteration $\quad(k=1,2,3, \mathrm{~K}) \quad$ approximate solutions $S^{1}, S^{2}, \mathrm{~K}, S^{n}, \mathrm{~K}$

But this requires firstly a choice of the initial condition $S^{0}$. Thereafter the sought solution $S$ to the problem (49) will be gotten by:

$$
S=\lim _{k \rightarrow \infty} S^{k} \text { if } S^{k} \text { converges }
$$

It was noted that the scheme (SBA) is a canonical form of Adomian. In this case, the Adomian algorithm can be written:

$$
\left\{\begin{array}{c}
S_{0}^{k}=S^{k}(0, Z)+S^{k}(a, t)-S^{k}(b, t)+\int_{0}^{t} N\left(S^{k-1}(u, Z)\right) d u-\frac{1}{\lambda} \iint_{a}^{b} \frac{\partial S^{k-1}(t, V)}{\partial t} d V d V+ \\
+\frac{1}{\lambda} \int_{a}^{b} \int_{a}^{b} N\left(S^{k-1}(t, V)\right) d V d V \\
S_{n}^{k}=\lambda \int_{0}^{t} \frac{\partial^{2} S_{n-1}^{k}(u, Z)}{\partial Z^{2}} d u
\end{array}\right.
$$

Where,

$$
\begin{aligned}
& N\left(S^{k-1}(t, V)\right)=\frac{K_{s} \omega}{c} \frac{\partial\left(S^{k-1}\right)^{n}}{\partial Z} \frac{\partial S^{k-1}}{\partial Z}+\frac{1}{c} \frac{\partial \varphi\left(S^{k-1}\right)}{\partial Z} \frac{\partial S^{k-1}}{\partial Z}- \\
& -\frac{1}{c}\left(K_{s} \omega\left(S^{k-1}\right)^{n}+\varphi\left(S^{k-1}\right) \frac{\partial^{2} S^{k-1}}{\partial Z^{2}}\right)- \\
& -\frac{1}{c h_{g}} \frac{\partial \varphi\left(S^{k-1}\right)}{\partial Z}
\end{aligned}
$$

And

$$
N\left(S^{k-1}(u, Z)\right)=-g\left(S^{k-1}\right) \frac{\partial S^{k-1}}{\partial t}-h\left(S^{k-1}\right) \frac{\partial S^{k-1}}{\partial t}
$$

In relation (70), one can ask:

$$
N\left(S^{k-1}\right)=\int_{0}^{t} S^{k-1}(u, Z) d u-\frac{1}{\lambda} \int_{a}^{b} \int_{a}^{b} \frac{\partial S^{k-1}(t, V)}{\partial t} d V d V+\frac{1}{\lambda} \int_{a}^{b} \int_{a}^{b} S^{k-1}(t, V) d V d V
$$

The various stages of resolution

Step 1: Calculation $S^{1}$

For $k=1$,

$N\left(S^{0}\right)=\int_{0}^{t} S^{0}(u, Z) d u-\frac{1}{\lambda} \int_{a}^{b} \int_{a}^{b} \frac{\partial S^{0}(t, V)}{\partial t} d V d V+\frac{1}{\lambda} \int_{a}^{b} \int_{a}^{b} S^{0}(t, V) d V d V$

Assuming that there is a $S^{0}$ such as $N\left(S^{0}\right)=0$.

$S^{1}$ Is solution of?

$S^{1}(t, Z)=S^{1}(a, t)-S^{1}(b, t)+S^{1}(0, Z)+\lambda \int_{0}^{t} \frac{\partial^{2} S^{1}(u, Z)}{\partial Z^{2}} d u$

So the classical Adomian algorithm is written

$$
\left\{\begin{array}{c}
S_{0}^{1}=S^{1}(a, t)-S^{1}(b, t)+S^{1}(0, Z) \\
S_{n}^{1}=\lambda \int_{0}^{t} \frac{\partial^{2} S_{n-1}^{1}(u, Z)}{\partial Z^{2}} d u \quad n=1,2, \Lambda
\end{array}\right.
$$

In detail, the description of the algorithm is as follows:

First step: Calculate of $S^{1}$

$\left\{\begin{array}{ccr}S_{0}^{1} & 1^{s t} & \text { term.of..the..series.of ..Adomian.in..step } 1 \\ S_{1}^{1} & 2^{\text {nd }} & \text { term.of ..series.of ..Adomian.in..step } 1 \\ S_{2}^{1} & 3^{r d} & \text { term.of .the..series.of ..Adomian..in..step } 1 \\ \mathrm{M} & \mathrm{M} & \mathrm{M} \\ S_{n}^{1} & (n+1)-t h & \text { term..of..the..series.of..Adomian.in..step } 1\end{array}\right.$

The approximate solution of this first stage is:

$$
S^{1}=\sum_{n=0}^{+\infty} S_{n}^{1}
$$

Second Step: Calculation of $S^{2}$ 
$\left\{\begin{array}{ccc}S_{0}^{21} & 1^{\text {st }} & \text { term.of..the..series.of ..Adomian.in..step2 } \\ S_{1}^{2} & 2^{\text {nd }} & \text { term.of..series.of..Adomian.in..step2 } \\ S_{2}^{21} & 3^{r d} & \text { term..of..the..series.of..Adomian.in..step2 } \\ \mathrm{M} & \mathrm{M} & \mathrm{M} \\ S_{n}^{21} & (n+1)-t h & \text { term.of..the..series.of..Adomian.in..step2 }\end{array}\right.$

The approximate solution of this second stage is written:

$$
S^{2}=\sum_{n=0}^{+\infty} S_{n}^{2}
$$

K-th stage: Calculation of $S^{k}$

$$
\left\{\begin{array}{ccc}
S_{0}^{1} & 1^{s t} & \text { term..of..the..series.of..Adomian.in..stepk } \\
S_{1}^{k} & 2^{\text {nd }} & \text { term.of ..series.of..Adomian.in..stepk } \\
S_{2}^{k} & 3^{r d} & \text { term.of..the..series.of..Adomian.in..stepk } \\
\mathrm{M} & \mathrm{M} & \mathrm{M} \\
S_{n}^{k} & (n+1)-t h & \text { term.of..the..series.of..Adomian.in..stepk }
\end{array}\right.
$$

The approximate solution of the K-th stage is:

$$
S^{k}=\sum_{n=0}^{+\infty} S_{n}^{k}
$$

The solution of the problem is obtained by:

$$
S=\lim _{k \rightarrow+\infty} S^{k}=\lim _{k \rightarrow+\infty}\left(\sum_{n=0}^{\infty} S_{n}^{k}\right)
$$

Possible choice of $S^{0}$

Here, we show how to choose the first iteration term $S^{0}$ the pattern of successive approximations for algorithms that converge faster to the exact solution sought by simplifying calculations. Just choose $S^{0}$ such as $N\left(S^{0}\right)=0$. This choice in fact, at the first iteration, only solves a linear problem.

\subsubsection{Practices application of SBA and simulation}

In this part, a model problem will be solved by our approach where parameters such as: $S^{1}(a, t), S^{1}(b, t)$ and $S^{1}(0, z)$ will set arbitrarily and parameters related to the model of Richards and the initial condition will be simulated. This will allow us to observe the different solutions and then compare the results to the solutions obtained in the same manner by other methods.

Either the system as follows:

$$
\left\{\begin{array}{c}
S_{0}^{1}=S^{1}(a, t)-S^{1}(b, t)+S^{1}(0, z) \\
S_{n}^{1}=\lambda \int_{0}^{t} \frac{\partial^{2} S_{n-1}^{1}(u, z)}{\partial z^{2}} d u
\end{array}\right.
$$

At this level, the issue was clearly set initial and boundary conditions by acting on the following parameters: $S^{1}(a, t), S^{1}(b, t)$ et $S^{1}(0, z)$. These parameters are set in a random manner by considering just the type of linear functions or combinations of these functions to highlight our method in this case of Richards equation transformed.

We set

$$
S^{1}(a, t)=\alpha, S^{1}(b, t)=\beta, S^{1}(0, z)=m+f(z),
$$

$0 \leq z \leq 100,0 \leq t \leq T$

With

$a=0, b=100$

$(\alpha, \beta) \in\left(\mathfrak{R}^{*}\right)^{2},(\mu, m) \in\left(\mathfrak{R}^{*}\right)^{2}, \ldots f(z)=e^{\mu z}$

Then

$S_{0}^{1}=S^{1}(a, t)-S^{1}(b, t)+S^{1}(0, z)=\alpha-\beta+e^{\mu z}$

So the system becomes:

$\left\{\begin{array}{c}S_{0}^{1}=\alpha-\beta+m+e^{\mu z} \\ S_{n}^{1}=\lambda \int_{0}^{t} \frac{\partial^{2} S_{n-1}^{1}}{\partial z^{2}} d u\end{array}\right.$

For

$n=1$

And

$k=1$

$S_{1}^{1}=\lambda \int_{0}^{t} \frac{\partial^{2} S_{0}^{1}}{\partial z^{2}} d u=\lambda \int_{0}^{t} \frac{\partial}{\partial z}\left[\frac{\partial}{\partial z}\left(\alpha-\beta+e^{\mu z}\right)\right] d u$

$S_{1}^{1}=\lambda \mu^{2} e^{\mu z} t \Rightarrow S_{1}^{1}=\left(\lambda \mu^{2} t\right) e^{\mu z}$

For

$n=2$

And

$k=1$

$S_{2}^{1}=\lambda \int_{0}^{t} \frac{\partial^{2} S_{1}^{1}}{\partial z^{2}} d u=\lambda \int_{0}^{t} \frac{\partial}{\partial z}\left[\frac{\partial}{\partial z}\left(\mu^{2}(\lambda t) e^{\mu z}\right] d u\right.$

$S_{2}^{1}=\left(\lambda \mu^{2} t\right)^{2} e^{\mu z}$

For

$n=3$

And

$k=1$

$S_{3}^{1}=\lambda \int_{0}^{t} \frac{\partial S_{2}^{1}}{\partial z^{2}} d u=\lambda \int_{0}^{t} \frac{\partial}{\partial z}\left[\frac{\partial}{\partial z}\left(e^{\mu z}\left(\lambda \mu^{2} t\right)^{2}\right] d u\right.$

$S_{3}^{1}=\left(\lambda \mu^{2} t\right)^{3} e^{\mu z}$

$\mathrm{N}$

Gradually therefore 


$$
\begin{aligned}
& S^{1}=\sum_{n=0}^{+\infty} S_{n}^{1}=S_{0}^{1}+S_{1}^{1}+S_{2}^{1}+S_{3}^{1}+\Lambda \\
& S^{1}=\left[\alpha-\beta+m+e^{\mu k}\right]+\left[\left(\lambda \mu^{2} t\right) e^{\mu z}\right]+\left[\left(\lambda \mu^{2} t\right)^{2} e^{\mu z}\right]+\left[\left(\lambda \mu^{2} t\right)^{3} e^{\mu k}\right]+\Lambda \\
& S^{1}=\alpha-\beta+m+\left[\left(\lambda \mu^{2} t\right)^{0}+\left(\lambda \mu^{2} t\right)^{1}+\left(\lambda \mu^{2} t\right)^{2}+\left(\lambda \mu^{2} t\right)^{3}+\Lambda\right] e^{\mu z} \\
& S^{1}=\alpha-\beta+m+\left[\sum_{n=0}^{+\infty}\left(\lambda \mu^{2} t\right)^{n}\right] e^{\mu z}=\alpha-\beta+m+\left[\sum_{n=0}^{+\infty}\left(\lambda \mu^{2} t\right)^{n}\right] e^{\mu z}
\end{aligned}
$$

As

$\sum_{n=0}^{+\infty}\left(\lambda \mu^{2} t\right)^{n}=\lim _{n \rightarrow+\infty} \frac{1-\left(\lambda \mu^{2} t\right)^{n+1}}{1-\left(\lambda \mu^{2} t\right)}=\frac{1}{1-\lambda \mu^{2} t}$

If

$\left|\lambda \mu^{2} t\right| \pi 1$

Than

$$
S^{1}=\alpha-\beta+m+\frac{e^{\mu z}}{1-\lambda \mu^{2} t}
$$

For

\section{$k \geq 2$}

We obtain in each step the same solution.

So

$$
S^{1}=S^{2}=\Lambda=S^{k}=\alpha-\beta+m+\frac{e^{\mu z}}{1-\lambda \mu^{2} t}
$$

With

$$
\lambda=\frac{K_{s}}{c}
$$

And

$$
c=\frac{\theta_{s}(2-n)}{h_{g}}
$$

As

$$
S(t, z)=\frac{\Psi(t, z)}{h_{g}} \Rightarrow \Psi(t, z)=h_{g} S(t, z)
$$

Then

$$
\Psi(t, z)=h_{g}\left[\alpha-\beta+m+\frac{e^{\mu z}}{1-\lambda \mu^{2} t}\right]
$$

As

$$
\Psi(t, z)=|h(t, z)|=-h(t, z) \ldots \ldots . . i f \ldots h \pi 0
$$

$$
h(t, z)=-h_{g}\left[\alpha-\beta+m+\frac{e^{\mu z}}{1-\lambda \mu^{2} t}\right]
$$

With

$$
\lambda=\frac{K_{s}}{c} \text { and } c=\frac{\theta_{s}(2-n)}{h_{g}}
$$

- $\theta_{s}:$ the water content in natural saturation,

- $K_{s}$ : the saturated hydraulic conductivity.

- $m$ and $n$ parameters relevant to the soil structure, $m=1-\frac{1}{n}$

- $h_{g}$ : the inflection point of the curve retention $h=f(\theta)$

- Note:

- Just simulate values $\alpha, \beta, m, \mu$.

- It remains to compare the solutions of the problem obtained with this method with the solutions of the problem obtained by other methods.

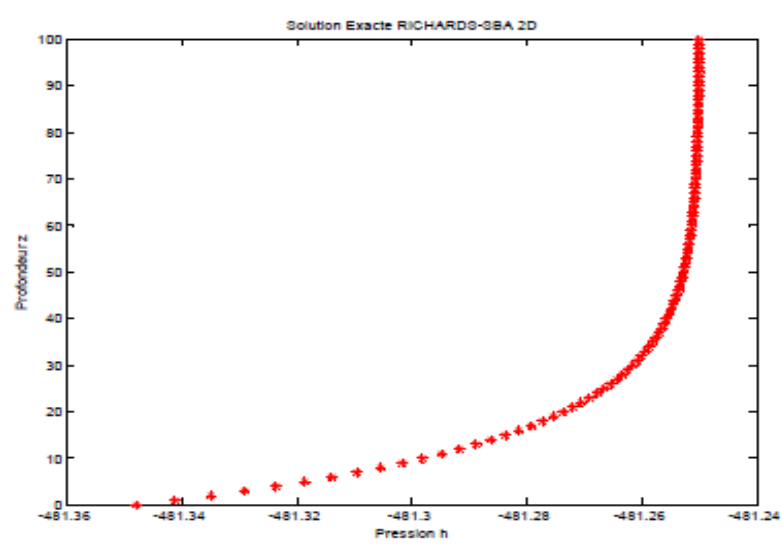

Fig. 1: $T=2 h$.

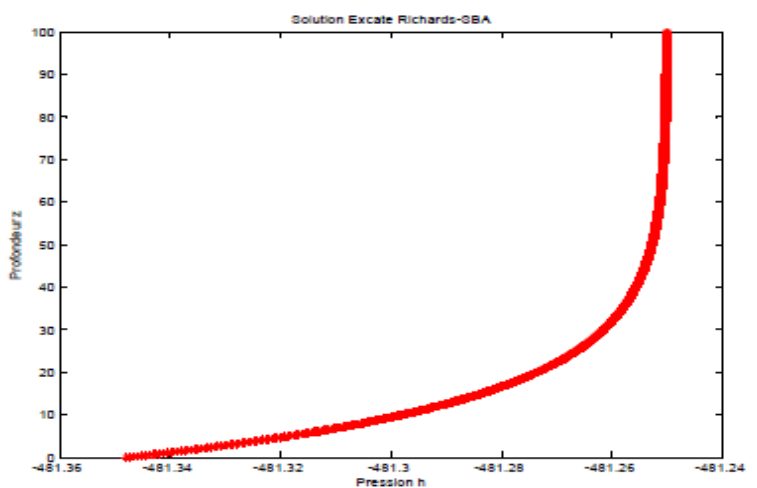

Fig. 2: $\mathrm{T}=3 \mathrm{~h}$.

Then 


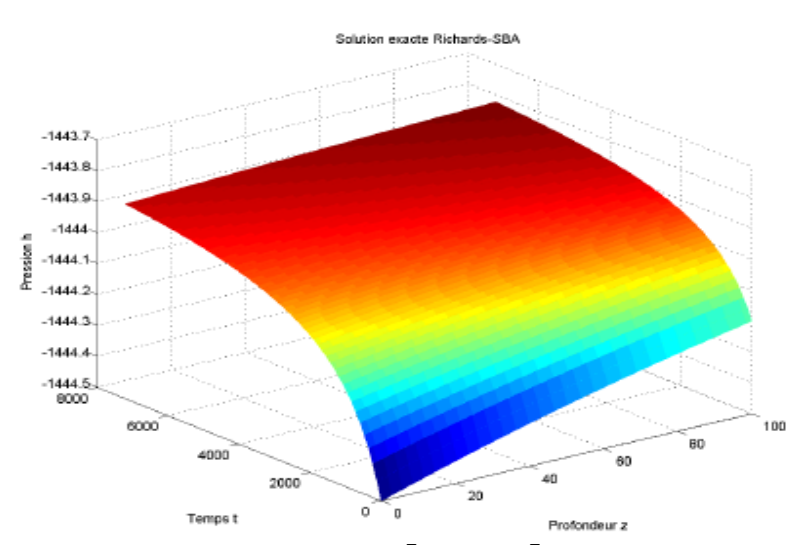

Fig. 3: $t \in[0,7200]$

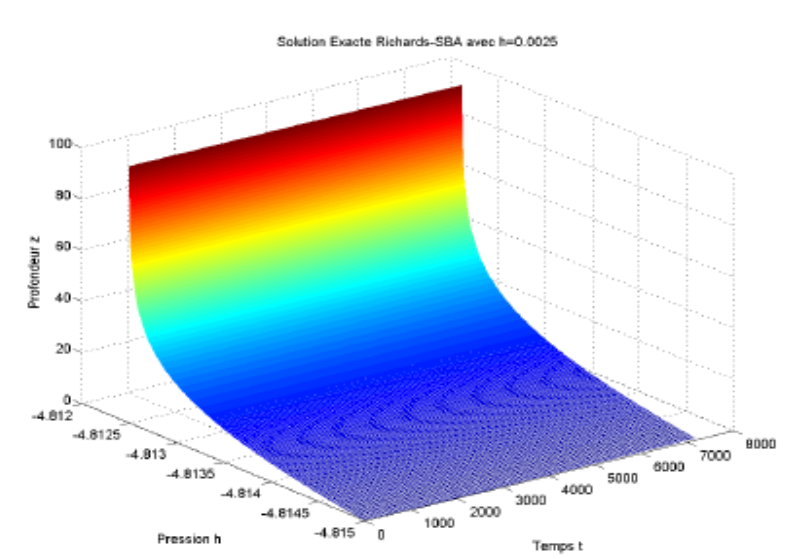

Fig.4: $t \in[0,7200]$.

\section{Interpretation}

On the curves in $2 d$ and $3 d$, we see that the pressure " $h$ " increases when depth " $z$ " increases. This proves that the developed technique and the solution obtained by this approach reflect «reality». This curve is obtain for $\mathrm{h}=0,25$ and $\mathrm{h}=0,0025$ in $2 \mathrm{D}$ and $3 \mathrm{D}$.

\section{Conclusion}

With this technic we could analytically solve Richards' equation through its modified form in which all the parameters of the initial model are preserved and it has been possible thanks to the technic of penalization applied to the initial function in SBA algorithm. Our approach is highly reliable because, whichever numerical method is used the solution can easily be compared to our solution according to the results of simulation obtained. Moreover, the constraints related to convergence, stability problems and that of taking into account of the nonlinearities were easily treated. We conclude that our argumentation is an innovation related to the resolution of the equation of Richards and is likely to be used to quantify the infiltrated water quantity which can breach the deep tablecloth of Bangui and its suburbs.

\section{References}

[1] A.Barari, M. Omidvar, A.R.Ghotbi, and D.D.Ganji. Numerical Analysis of Richard's problem for water penetration in unsatured soils. Hydrology and Earth System Sciences Discussions. 6, 6359-6885, 2009. https://doi.org/10.5194/hessd-6-6359-2009.

[2] P.Ngnepieba, F.X. Le Dimet, A; Boukong, and G. Nguetseng. Identification des parametres: une application à l'équation de Richards. ARIMA Vol1-127-157, 2002.
[3] B.Abbo, B.Some, Nouvel algorithme numérique de résolution des équations différentielles ordinaires(EDO) et des équations aux dérivées partielles(EDP) non linéaires.119p. Mathématiques appliquées. Ouagadougou : Université d'Ouagadougou, 2007.

[4] S.Bisso, B.Some, Résolution et simulation numérique d'un problème de contrôle optimal gouverné par des équations aux dérivées partielles de type diffusion-réaction issues de la modélisation mathématique en traitement du cancer du cerveau. 164p. Mathématiques appliquées. Ouagadougou : Université d'Ouagadougou, 2003.

[5] D.Crevoisier, J.C.Mailhol, Modélisation analytique des transferts biet tridirectionnels eau-soluté : Application à l'irrigation à la raie et à la micro-irrigation. 263p. Sciences de l'eau. Paris : Ecole Nationale du Génie Rural, des Eaux et Forêts, 2005.

[6] S. Kaskassian., J.-P. GaudeT, J. Chastanet, F. Decung, R. Angulojaramillo, S. Szenknect, J.-M. Côme, D. Getto, V barthes, M krimissa 2009. Projet ANRPRECODD/ TRANSAT 2005-2009, Evaluation des temps de Transfert, dans la zone Non Saturée des sols, de contaminants dissous ou particulaires, Guide technique, $106 \mathrm{p}$.

[7] W.O. Sawadogo, M. N. Alla, Modélisation hydrogéologique : Ecoulement en milieux poreux fracturés, problème inverse et transfert de polluants. 154p. Mathématiques appliquées. Ouagadougou : Université d'Ouagadougou, 2012.

[8] F Lafolie, C.Thirriot, Etude numérique de la résolution des équations de transfert : Application à l'irrigation localisée.260p. Sciences du sol. Avignon : Université d'Avignon, 1986.

[9] Blaise Some «Optimisation générale et Méthodes numériques » notes de cours DEA / Mathématique. Université d'Ouagadougou. 2010 .

[10] A.Mermoud «Etat de l'eau du sol » notes de cours / Cours de physique du sol. Ecole Polytechnique de Lausanne. 2006. Y. Pare, Résolution de quelques équations

[11] fonctionnelles par la numérique SBA (SOME BLAISE-ABBO), Thèse de Doctorat unique. Université d'Ouagadougou, mai 2010, UFR/SEA, Département Mathématiques et Informatique (Burkina Faso).

[12] F. Bassono- Etude de quelques équations fonctionnelles par les méthodes : Some Blaise Abbo, décompositionnelle d.Adomian et perturbations. Université d'Ouagadougou, Janvier 2013, UFR/SEA, Département Mathématiques et Informatique (Burkina Faso).

[13] C.L.Djebebe et al. Characterisation of the aquifers of the Bangui Urban area, Central African Republic, as alternative drinking water supply resource, Hydrological Sciences Journal, 58(8) 2013.

[14] R.Yaro- Contribution à la résolution de quelques modèles mathématiques de la dynamique des populations par les méthodes d'Adomian, SBA et des Perturbations. Université d'Ouagadougou, Mai 2016, UFR/SEA, Département Mathématiques et Informatique (Burkina Faso). 